\title{
Reinvestigation of piezooptic anisotropy appearing in a crystalline disk loaded along its diameter
}

\author{
Savaryn V., Krupych O., Skab I. and Vlokh R. \\ Institute of Physical Optics, 23 Dragomanov Street, 79005 Lviv, Ukraine, \\ e-mail: vlokh@ifo.lviv.ua
}

Received: 26.03 .2014

\begin{abstract}
In order to correct the errors present in our recent study (Savaryn V. et al. Ukr. J. Phys. Opt. 13 (2012) 82), we have rederived the main phenomenological relations describing the changes in optical anisotropy of crystalline disks loaded along their diameters. Basing on the analysis of these relations, we have developed an improved technique for determining the piezooptic coefficients $\pi_{44}, \pi_{55}$ and $\pi_{66}$ for the crystals of almost all of the point symmetry groups. The technique is based on studying the spatial distributions of optical birefringence and optical indicatrix rotation angle induced along the chords and diameters of a crystalline disk compressed along its diameter.
\end{abstract}

Keywords: piezooptic effect, 2D stress state, crystals, crystalline disk

PACS: $78.20 . \mathrm{Hp}, 78.20 . \mathrm{Ci}$

UDC: $535.55+53.082 .5$

\section{Introduction}

It is well known that piezooptic effect consists in changes of the optical impermeability coefficients $\Delta B_{i j}$ (or the refractive indices $B_{i j}=B_{k}=\left(1 / n^{2}\right)_{k}$ ) of a material medium, which appear under the action of mechanical stresses $\sigma_{n l}=\sigma_{m}$. This is described by the phenomenological relation

$$
\Delta B_{k}=\pi_{k m} \sigma_{m}
$$

where $\pi_{k m}$ denotes a fourth-rank piezooptic tensor [1]. The effect is usually studied when uniaxially loading parallelepiped-shaped samples. Unfortunately, this leads to appearance of uncontrollable spatially inhomogeneous stresses inside a sample and, as a result, to dramatic lowering of the accuracy for the piezooptic coefficients.

Recently we have demonstrated that application of inhomogeneous 2D stresses, which are known in advance, yields in a novel technique for determination of the piezooptic coefficients. Moreover, this technique offers a number of advantages, e.g. the piezooptic coefficients can be determined with high enough accuracy, when applying torsion stresses $[2,3]$ or four-point bending stresses [4] to crystalline samples. In particular, in our work [5] we have reported a specific technique developed for measuring the piezooptic coefficients $\pi_{k k}(k=4,5,6)$ for the crystals that belong to almost all of the point groups of symmetry. It is based on the studies of spatial distributions of optical birefringence and optical indicatrix rotation along different diameters of a crystalline disk which is compressed along one of its diameter.

Besides, phenomenological relations have been presented in Ref. [5] that describe the induced piezooptic changes occurring in the optical birefringence and the optical indicatrix 
rotation angle. Unfortunately, these relations have been derived using incorrect underlying expressions for the shear stress tensor components $\sigma_{4}, \sigma_{5}$ and $\sigma_{6}$ that appear due to compression of the disks. The latter fact has also led to partially incorrect conclusions drawn in Ref. [5]. First of all, this is the finding that the piezooptic coefficients $\pi_{k k}$ can be determined when the optical beam is scanned along the disk's diameters parallel or perpendicular to the loading force. In the present work we have rederived the main theoretical relations that describe the induced changes in the optical birefringence and the optical indicatrix rotation for the crystalline disks loaded along their diameters. We have also verified and improved the main conclusions of the work [5].

\section{General phenomenological relations}

It is known $[6,7]$ that loading of a disk along its diameter produces a non-uniform spatial distribution of mechanical stresses. Let us introduce a coordinate system $X Y Z$ associated with the eigenvectors of optical impermeability tensor that characterizes a given crystalline material. Suppose that a $Z$-cut crystalline disk is loaded along its diameter parallel to $Y$ axis. Then the loading force has the only component $P=P_{2}$, while the stress tensor components $\sigma_{1}, \sigma_{2}$ and $\sigma_{6}$ will be equal to

$$
\begin{aligned}
& \sigma_{1}=-\frac{2 P_{2}}{\pi d}\left[\frac{(R-Y) X^{2}}{\left\{X^{2}+(R-Y)^{2}\right\}^{2}}+\frac{(R+Y) X^{2}}{\left\{X^{2}+(R+Y)^{2}\right\}^{2}}-\frac{1}{2 R}\right], \\
& \sigma_{2}=-\frac{2 P_{2}}{\pi d}\left[\frac{(R-Y)^{3}}{\left\{X^{2}+(R-Y)^{2}\right\}^{2}}+\frac{(R+Y)^{3}}{\left\{X^{2}+(R+Y)^{2}\right\}^{2}}-\frac{1}{2 R}\right], \\
& \sigma_{6}=\frac{2 P_{2}}{\pi d}\left[\frac{(R-Y)^{2} X}{\left\{X^{2}+(R-Y)^{2}\right\}^{2}}-\frac{(R+Y)^{2} X}{\left\{X^{2}+(R+Y)^{2}\right\}^{2}}\right] .
\end{aligned}
$$

Here $d$ denotes the thickness of the disk and $R$ its radius.

The mechanical stresses appearing along the diameter parallel to $X$ axis (i.e., in the case of $Y=0)$ and along the diameter parallel to $Y$ axis $(X=0)$ are described respectively by the relations

$$
\sigma_{1}(X)=-\frac{2 P_{2}}{\pi d}\left[\frac{2 R X^{2}}{\left(X^{2}+R^{2}\right)^{2}}-\frac{1}{2 R}\right], \sigma_{2}(X)=-\frac{2 P_{2}}{\pi d}\left[\frac{2 R^{3}}{\left(X^{2}+R^{2}\right)^{2}}-\frac{1}{2 R}\right], \sigma_{6}(X)=0
$$

and

$$
\sigma_{1}(Y)=\frac{P_{2}}{\pi d R}, \sigma_{2}(Y)=-\frac{2 P_{2}}{\pi d}\left[\frac{2 R}{R^{2}-Y^{2}}-\frac{1}{2 R}\right], \sigma_{6}(Y)=0 .
$$

As a consequence, we have the equality $\sigma_{6}=0$ along the both diameters mentioned above. At the same time, the stress tensor components $\sigma_{1}, \sigma_{2}$ and $\sigma_{6}$ remain nonzero along the chords which are parallel to the $X$ or $Y$ axes (i.e., when $Y$ or $X$ coordinates are equal to $R / 2$ ). These components read as 


$$
\begin{aligned}
& \sigma_{1}(X)=-\frac{P_{2}}{\pi d}\left[\frac{R X^{2}}{\left(X^{2}+R^{2} / 4\right)^{2}}+\frac{3 R X^{2}}{\left(X^{2}+9 R^{2} / 4\right)^{2}}-\frac{1}{R}\right], \\
& \sigma_{2}(X)=-\frac{P_{2}}{\pi d}\left[\frac{R^{3}}{4\left(X^{2}+R^{2} / 4\right)^{2}}+\frac{27 R^{3}}{4\left(X^{2}+9 R^{2} / 4\right)^{2}}-\frac{1}{R}\right], \\
& \sigma_{6}(X)=\frac{P_{2}}{2 \pi d}\left[\frac{R^{2} X}{\left(X^{2}+R^{2} / 4\right)^{2}}-\frac{9 R^{2} X}{\left(X^{2}+9 R^{2} / 4\right)^{2}}\right]
\end{aligned}
$$

or

$$
\begin{aligned}
& \sigma_{1}(Y)=-\frac{P_{2}}{\pi d}\left[\frac{(R-Y) R^{2}}{2\left\{(R-Y)^{2}+R^{2} / 4\right\}^{2}}+\frac{(R+Y) R^{2}}{2\left\{(R+Y)^{2}+R^{2} / 4\right\}^{2}}-\frac{1}{R}\right], \\
& \sigma_{2}(Y)=-\frac{2 P_{2}}{\pi d}\left[\frac{(R-Y)^{3}}{\left\{(R-Y)^{2}+R^{2} / 4\right\}^{2}}+\frac{(R+Y)^{3}}{\left\{(R+Y)^{2}+R^{2} / 4\right\}^{2}}-\frac{1}{2 R}\right], \\
& \sigma_{6}(Y)=\frac{P_{2}}{\pi d}\left[\frac{(R-Y)^{2} R}{\left\{(R-Y)^{2}+R^{2} / 4\right\}^{2}}-\frac{(R+Y)^{2} R}{\left\{(R+Y)^{2}+R^{2} / 4\right\}^{2}}\right],
\end{aligned}
$$

respectively. Similar relations may easily be written for the $X$ - and $Y$-cuts of the crystalline disk. Using all of those formulae, we have rederived the expressions that describe the induced optical birefringence and the angle of optical indicatrix rotation for the loaded disks made of crystals of all point symmetry groups (see Table 1).

Using the above equations valid for the crystals of different symmetries, we will analyze them and, in this way, find the experimental geometries for which the piezooptic coefficients $\pi_{k k}$ can be determined using the crystalline disks loaded along their diameters.

\subsection{Cubic crystals}

Basing on Eqs. (13)-(24) for the optical birefringence and the optical indicatrix rotation angle, one can determine the piezooptic coefficient $\pi_{44}$ for the cubic crystals in the experimental geometries where the shear stress tensor components $\sigma_{4}, \sigma_{5}$ and $\sigma_{6}$ are nonzero. As seen from Eqs. (9) and (12), these conditions are satisfied whenever the light beam is scanned along the chords parallel to the principal axes. For example, the induced birefringence and the induced optical indicatrix rotation are given by Eqs. (17) and (18) if a Z-cut disk made of crystals of the symmetry groups 432, $\overline{4} 3 \mathrm{~m}$ and $\mathrm{m} 3 \mathrm{~m}$ is loaded along its diameter parallel to the $Y$ axis. Employing Eq. (17) and the distributions of the induced birefringence measured experimentally along the chords at the height $Y=R / 2$ and $X=R / 2$, one can get the difference of the piezooptic coefficients $\pi_{11}-\pi_{12}$ and the coefficient $\pi_{44}$. Notice that the coefficient $\pi_{44}$ can also be determined from Eq. (18), using the experimental dependences of optical indicatrix rotation angle on the coordinates read along the directions of chords parallel to the $X$ and $Y$ axes. In addition, similar measurements can be carried out for the $X$ - and $Y$-cuts of crystalline disks. 


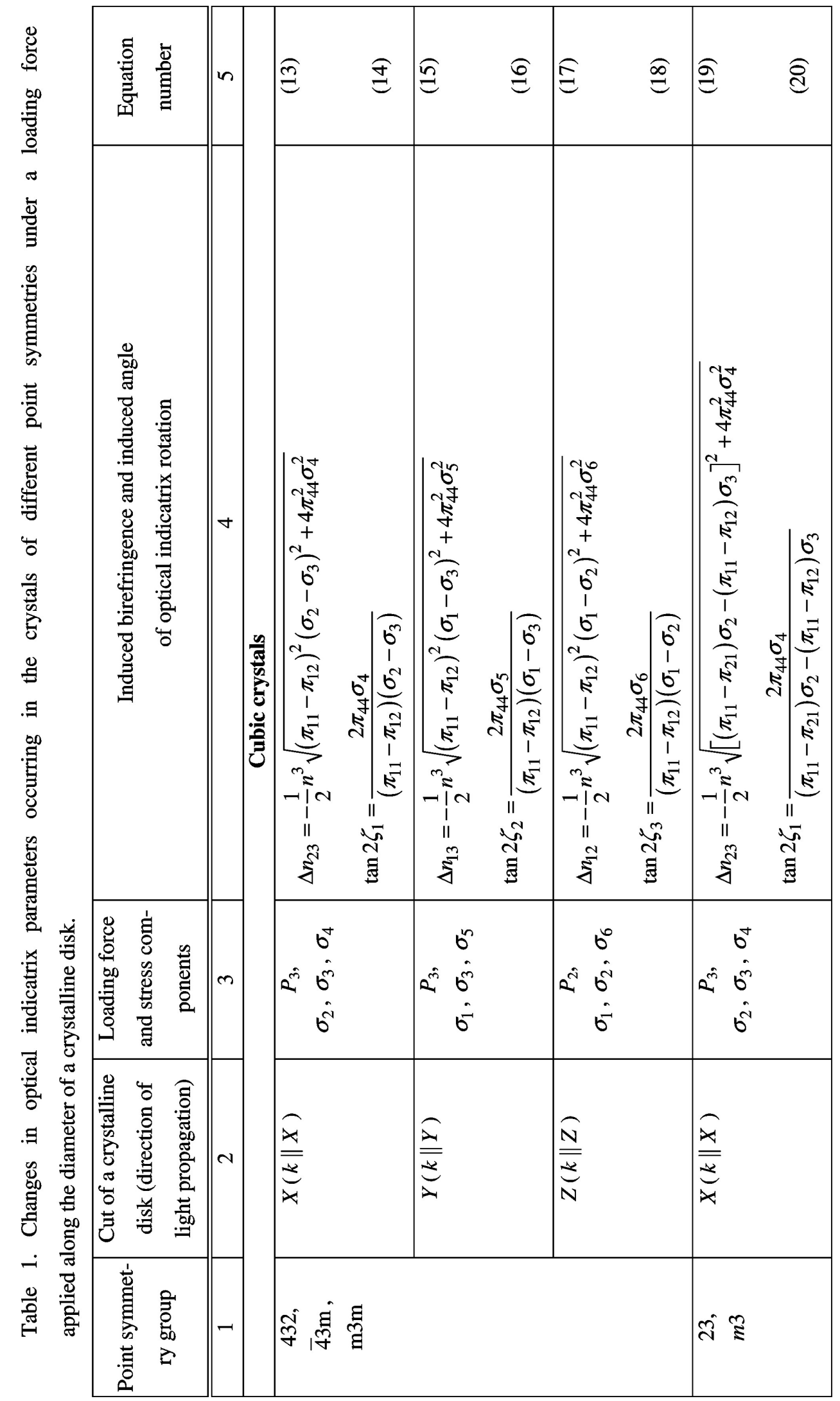

Ukr. J. Phys. Opt. 2014, Volume 15, Issue 2 
Savaryn V. et al

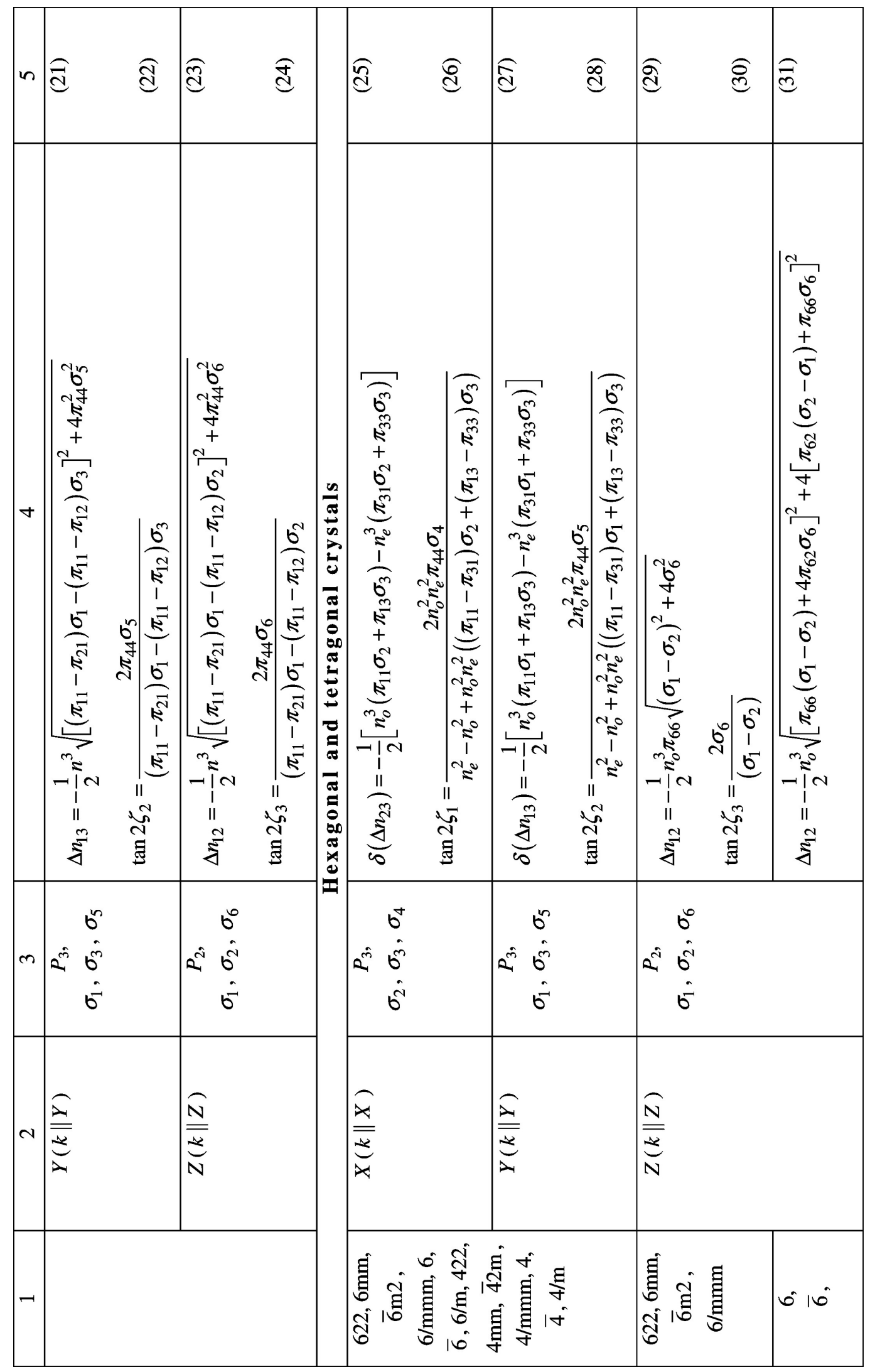




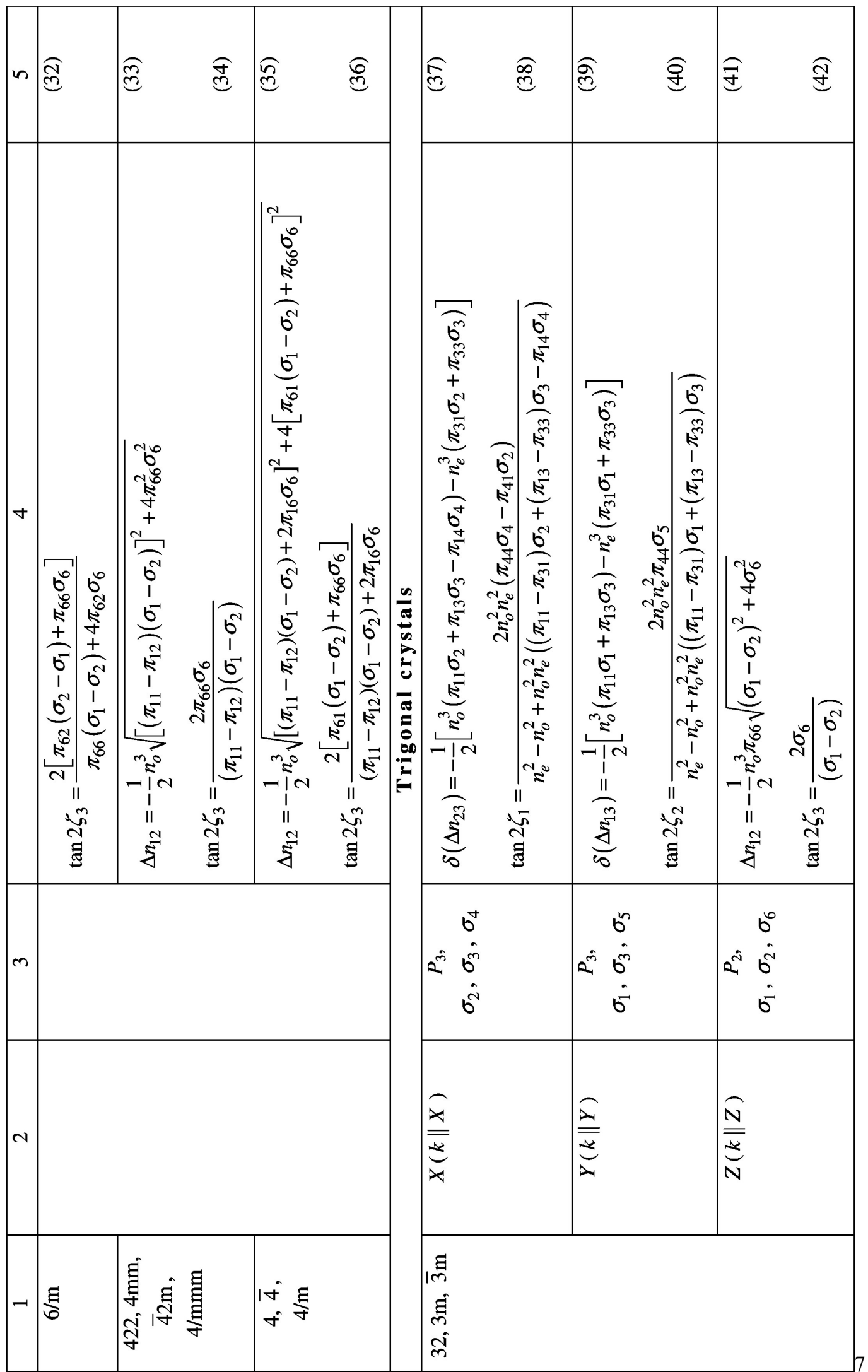

Ukr. J. Phys. Opt. 2014, Volume 15, Issue 2 


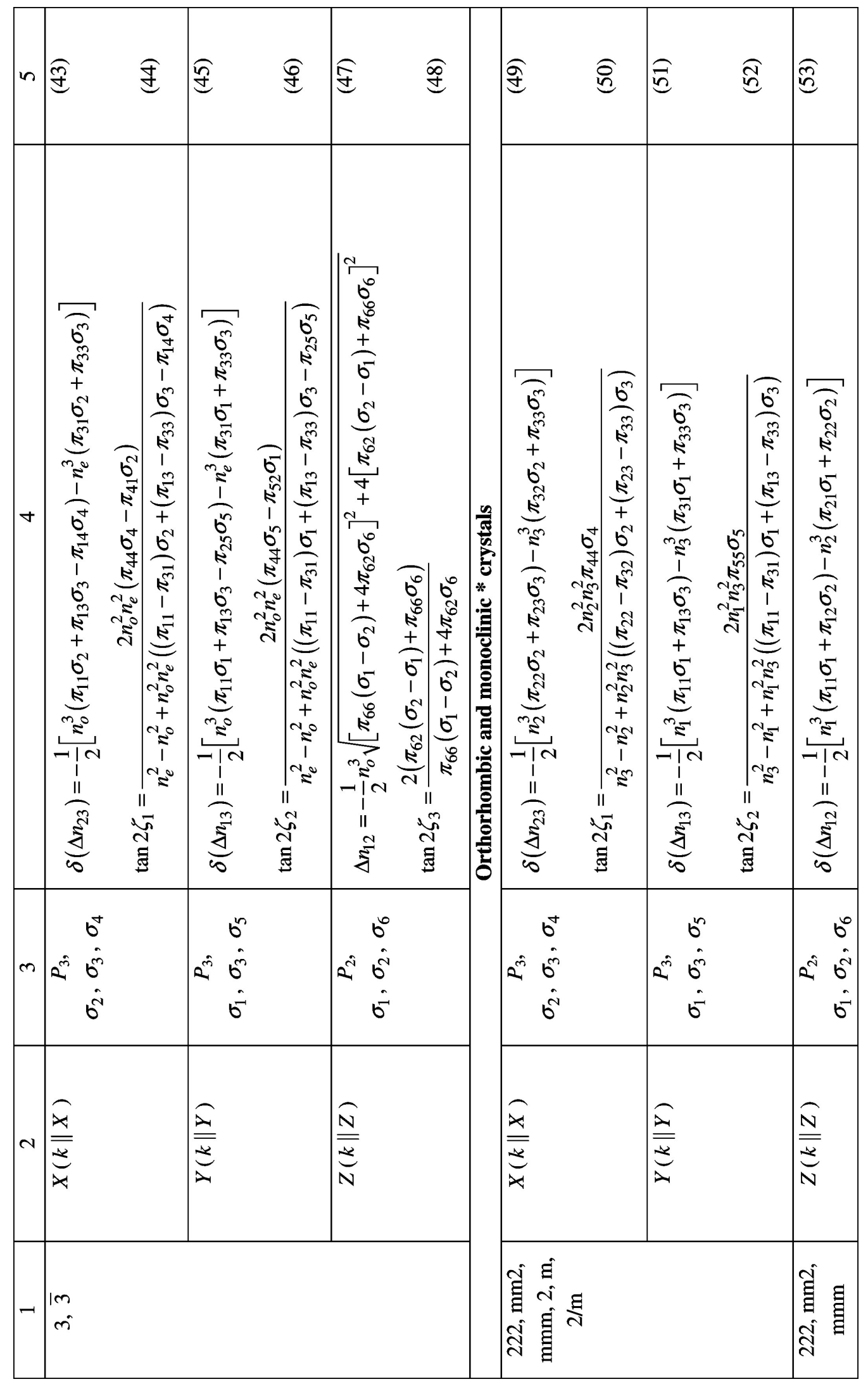




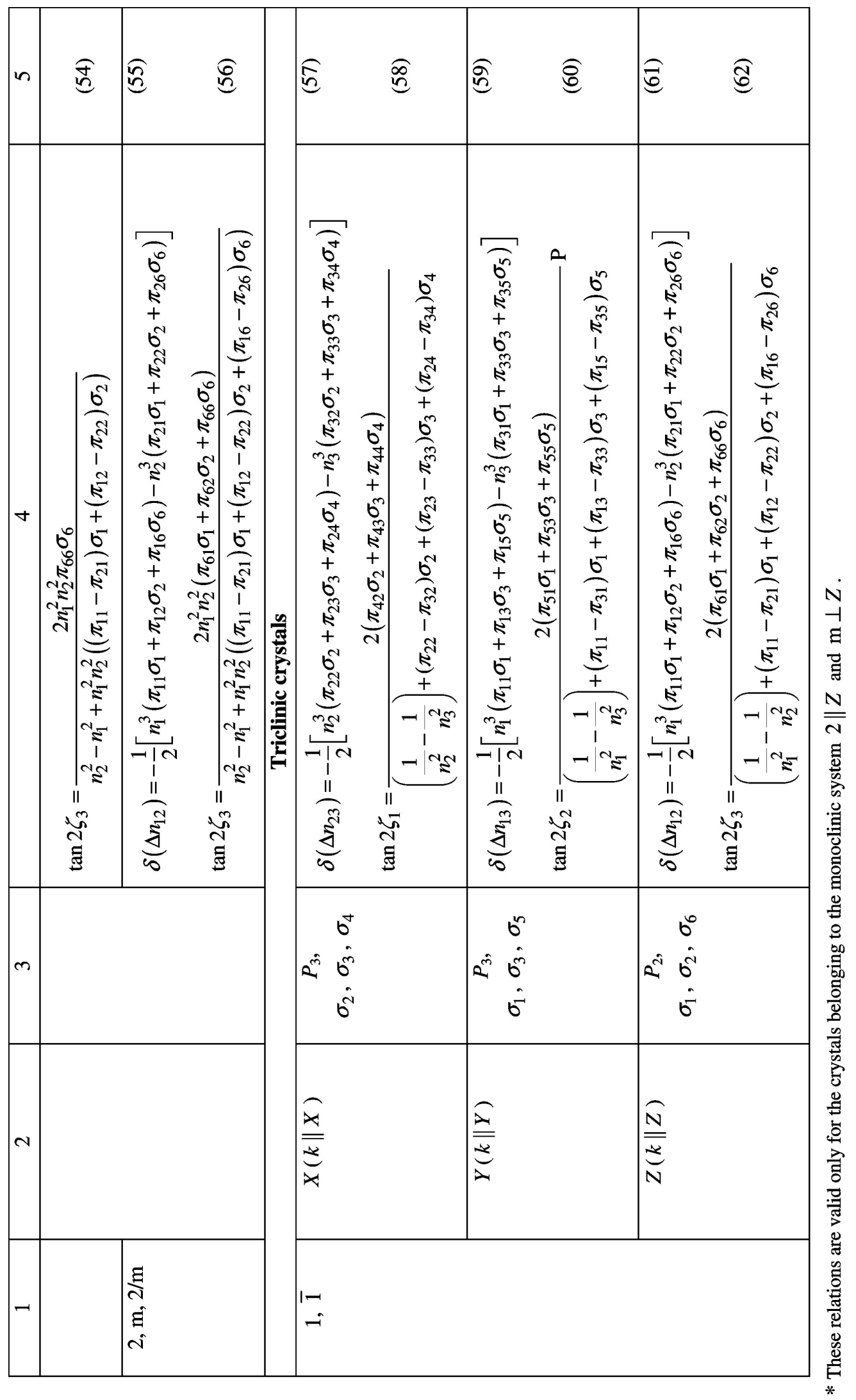

Ukr. J. Phys. Opt. 2014, Volume 15, Issue 2 
Under the same geometry (i.e., the $Z$-cut of a crystalline disk and the loading force $P_{2}$ ), the relation for the induced birefringence $\Delta n_{12}(X, Y)$ for the cubic crystals belonging to the groups 23 and $\mathrm{m} 3$ follows from Eq. (23) respectively under the conditions of $Y=0$ and $X=0$ :

$$
\begin{aligned}
& \Delta n_{12}(X, 0)=-\frac{1}{2} n^{3}\left[\left(\pi_{11}-\pi_{21}\right) \sigma_{1}(X)-\left(\pi_{11}-\pi_{12}\right) \sigma_{2}(X)\right], \\
& \Delta n_{12}(0, Y)=-\frac{1}{2} n^{3}\left[\left(\pi_{11}-\pi_{21}\right) \sigma_{1}(Y)-\left(\pi_{11}-\pi_{12}\right) \sigma_{2}(Y)\right] .
\end{aligned}
$$

Here $n$ denotes the initial (unperturbed) refractive index, and $\sigma_{1}(X), \sigma_{2}(X)$ and $\sigma_{1}(Y), \sigma_{2}(Y)$ are defined respectively by Eqs. (5) and (6). Then the induced birefringences along the chords $Y=R / 2$ and $X=R / 2$ are as follows:

$$
\Delta n_{12}(X, R / 2)=-\frac{1}{2} n^{3} \sqrt{\left[\left(\pi_{11}-\pi_{21}\right) \sigma_{1}(X)-\left(\pi_{11}-\pi_{12}\right) \sigma_{2}(X)\right]^{2}+4 \pi_{44}^{2} \sigma_{6}^{2}(X)}
$$

and

$$
\Delta n_{12}(R / 2, Y)=-\frac{1}{2} n^{3} \sqrt{\left[\left(\pi_{11}-\pi_{21}\right) \sigma_{1}(Y)-\left(\pi_{11}-\pi_{12}\right) \sigma_{2}(Y)\right]^{2}+4 \pi_{44}^{2} \sigma_{6}^{2}(Y)},
$$

Here $\sigma_{1}(X), \sigma_{2}(X), \sigma_{6}(X)$ and $\sigma_{1}(Y), \sigma_{2}(Y), \sigma_{6}(Y)$ are given respectively by Eqs. (7)-(9) and Eqs. (10)-(12).

Using these formulae, one can obtain the differences $\pi_{11}-\pi_{21}$ and $\pi_{11}-\pi_{12}$ of the piezooptic coefficients, as well as the piezooptic coefficient $\pi_{44}$. Notice that similar measurements can be performed in the other geometries, e.g. when the light propagates along the $X$ and $Y$ directions and the only loading component is $P_{3}$. Hence, the $\pi_{44}$ coefficient can indeed be easily measured for the cubic crystals. This agrees well with the conclusion drawn in Ref. [5], though the relevant practical procedures differ from those suggested in that work. Namely, for this aim one has to derive experimentally spatial distributions of the optical birefringence and the angle of optical indicatrix rotation along the chords.

\subsection{Hexagonal and tetragonal crystals}

For all of hexagonal and tetragonal crystals, Eqs. (27) and (28) (see Table 1) describe the birefringence and the optical indicatrix rotation angle induced by the force component $P_{3}$ in the $Y$ cut disks under the condition $X=R / 2$ (i.e., along the chords). Taking into account that the natural birefringence is relatively small (i.e., $n_{o} \simeq n_{e}=\bar{n}$ ), one can simplify these equations to the following form:

$$
\begin{aligned}
& \delta\left(\Delta n_{13}\right)=-\frac{1}{2} \bar{n}^{3}\left[\left(\pi_{11}-\pi_{31}\right) \sigma_{1}(Z)+\left(\pi_{13}-\pi_{33}\right) \sigma_{3}(Z)\right], \\
& \tan 2 \zeta_{2}=\frac{2 \pi_{44} \sigma_{5}(Z)}{\left(\pi_{11}-\pi_{31}\right) \sigma_{1}(Z)+\left(\pi_{13}-\pi_{33}\right) \sigma_{3}(Z)} .
\end{aligned}
$$

Let us insert the relation appearing in the square brackets of Eq. (67) into Eq. (68) and take into account that the mechanical stress component $\sigma_{5}(Z)$ is given by the formula

$$
\sigma_{5}(Z)=\frac{P_{3}}{\pi d}\left[\frac{(R-Z)^{2} R}{\left\{(R-Z)^{2}+R^{2} / 4\right\}^{2}}-\frac{(R+Z)^{2} R}{\left\{(R+Z)^{2}+R^{2} / 4\right\}^{2}}\right] .
$$


Then the coefficient $\pi_{44}$ can be obtained. Now we consider the disks that have their faces perpendicular to the $Z$ axis and are made of crystals belonging to the hexagonal symmetry groups $622,6 \mathrm{~mm}, \overline{6} \mathrm{~m} 2$ and $6 / \mathrm{mmm}$. The spatial $X Y$ distribution of the induced birefringence known from experiments enables determining the coefficient $\pi_{66}$ with the aid of Eq. (29).

Now we proceed to the spatial birefringence distributions induced along the chords $Y=R / 2$ and $X=R / 2$ in the $Z$-cut disks of crystals that belong to the point groups $6, \overline{6}$ and $6 / \mathrm{m}$ and are loaded by the force $P_{2}$. The appropriate relations may be derived using Eq. (31) and taking Eqs. (7)-(12) into account. Then solving the system of equations for $\Delta n_{12}(X, R / 2)$ and $\Delta n_{12}(R / 2, Y)$ facilitates experimental determination of the piezooptic coefficients $\pi_{62}$ and $\pi_{66}$. This is also true for the point symmetries $422,4 \mathrm{~mm}, \overline{4} 2 \mathrm{~m}$ and $4 / \mathrm{mmm}$, for which the induced birefringence is defined by Eq. (33). Then the coefficients $\pi_{11}-\pi_{12}$ and $\pi_{66}$ can be also determined.

For the crystals of tetragonal groups $4, \overline{4}$ and $4 / \mathrm{m}$, one can easily derive the birefringence induced for the case of the $Z$-cut crystalline disk and the $P_{2}$ force. Considering the experimental distributions of the induced birefringence along the four directions $(Y=0, X=0, Y=R / 2$ and $X=R / 2$ ) and solving the system of Eqs. (35) for these directions (i.e., the relations for $\Delta n_{12}(X, 0), \Delta n_{12}(0, Y), \Delta n_{12}(X, R / 2)$ and $\left.\Delta n_{12}(R / 2, Y)\right)$, one can find the coefficients $\pi_{16}$, $\pi_{61}, \pi_{66}$ and the combination $\pi_{11}-\pi_{12}$.

Hence, one can determine the coefficient $\pi_{44}$ for the crystals of hexagonal and tetragonal systems after spatial distributions of the induced birefringence and the angle of optical indicatrix rotation have been obtained along the chords but not the diameters as suggested in Ref. [5]. On the other hand, the coefficient $\pi_{66}$ can be determined using the experimental spatial distributions of the optical indicatrix parameters obtained along the chords for the symmetry groups $422,4 \mathrm{~mm}$, $\overline{4} 2 \mathrm{~m}, 4 / \mathrm{mmm}, 4, \overline{4}, 4 / \mathrm{m}, 6, \overline{6}$ and $6 / \mathrm{m}$, and along the diameters for the groups $622,6 \mathrm{~mm}, \overline{6} \mathrm{~m} 2$ and $6 / \mathrm{mmm}$. Some additional coefficients and their combinations can be determined, too.

\subsection{Trigonal crystals}

As follows from Eqs. (39) and (40), the coefficient $\pi_{44}$ for the crystals of trigonal groups 32, 3m and $\overline{3} \mathrm{~m}$ becomes measurable in the same way as for the case of hexagonal or tetragonal crystals. Here the birefringence and the optical indicatrix rotation induced by the force $P_{3}$ in the $Y$-cut disks under the condition $X=R / 2$ are described by Eqs. (39) and (40), which are identical respectively to Eqs. (27) and (28) (see Table 1). In addition, one can determine the coefficient $\pi_{66}$ from Eq. (41) and experimental $X Y$-distribution of the birefringence induced along the disk diameters. Then the $Z$-cut crystalline disk should be employed and the force $P_{2}$ should be applied.

Let us consider the crystals of the trigonal groups 3 and $\overline{3}$. Here the $\pi_{44}$ coefficient can be measured in the geometry given by $k \| Y$ and $P=P_{3}$. The birefringence and the optical indicatrix rotation angle induced by the force $P_{3}$ in the $Y$-cut disk are described by Eqs. (45) and (46) (see Table 1). Considering that $n_{o} \simeq n_{e}=\bar{n}$, we simplify the corresponding equations yielding in

$$
\delta\left(\Delta n_{13}\right)=-\frac{1}{2} \bar{n}^{3}\left[\left(\pi_{11}-\pi_{31}\right) \sigma_{1}(Z)+\left(\pi_{13}-\pi_{33}\right) \sigma_{3}(Z)-\pi_{25} \sigma_{5}(Z)\right],
$$




$$
\tan 2 \zeta_{2}(Z)=\frac{2\left(\pi_{44} \sigma_{5}(Z)-\pi_{52} \sigma_{1}(Z)\right)}{\left(\pi_{11}-\pi_{31}\right) \sigma_{1}(Z)+\left(\pi_{13}-\pi_{33}\right) \sigma_{3}(Z)-\pi_{25} \sigma_{5}(Z)}
$$

under the condition of $X=R / 2$, or

$$
\begin{aligned}
& \delta\left(\Delta n_{13}\right)=-\frac{1}{2} \bar{n}^{3}\left[\left(\pi_{11}-\pi_{31}\right) \sigma_{1}(X)+\left(\pi_{13}-\pi_{33}\right) \sigma_{3}(X)-\pi_{25} \sigma_{5}(X)\right], \\
& \tan 2 \zeta_{2}(X)=\frac{2\left(\pi_{44} \sigma_{5}(X)-\pi_{52} \sigma_{1}(X)\right)}{\left(\pi_{11}-\pi_{31}\right) \sigma_{1}(X)+\left(\pi_{13}-\pi_{33}\right) \sigma_{3}(X)-\pi_{25} \sigma_{5}(X)}
\end{aligned}
$$

when we have $Z=R / 2$. Here the mechanical stress tensor component $\sigma_{5}(Z)$ is given by Eq. (69), whereas the components $\sigma_{1}(Z), \sigma_{1}(X)$ and $\sigma_{5}(X)$ are defined as

$$
\begin{aligned}
& \sigma_{1}(Z)=-\frac{P_{3}}{\pi d}\left[\frac{(R-Z) R^{2}}{2\left\{(R-Z)^{2}+R^{2} / 4\right\}^{2}}+\frac{(R+Z) R^{2}}{2\left\{(R+Z)^{2}+R^{2} / 4\right\}^{2}}-\frac{1}{R}\right], \\
& \sigma_{1}(X)=-\frac{P_{3}}{\pi d}\left[\frac{R X^{2}}{\left(X^{2}+R^{2} / 4\right)^{2}}+\frac{3 R X^{2}}{\left(X^{2}+9 R^{2} / 4\right)^{2}}-\frac{1}{R}\right], \\
& \sigma_{5}(X)=\frac{P_{3}}{2 \pi d}\left[\frac{R^{2} X}{\left(X^{2}+R^{2} / 4\right)^{2}}-\frac{9 R^{2} X}{\left(X^{2}+9 R^{2} / 4\right)^{2}}\right] .
\end{aligned}
$$

Inserting the expressions appearing in the square brackets of Eqs. (70) and (72) into the denominators of respectively Eqs. (71) and (73), and solving the system of Eqs. (71) and (73), one can obtain the coefficients $\pi_{44}$ and $\pi_{52}$.

Thus, the coefficient $\pi_{66}$ for the symmetry groups $32,3 \mathrm{~m}$ and $\overline{3} \mathrm{~m}$ can be found while measuring spatial distributions of the optical indicatrix parameters along the disk diameters. It is this statement that has just been made in Ref. [5]. However, the coefficient $\pi_{44}$ for the trigonal crystals has to be derived using the same spatial distributions measured along the chords. The latter is also true of the $\pi_{66}$ coefficient for the symmetry groups 3 and $\overline{3}$.

\subsection{Orthorhombic, monoclinic and triclinic crystals}

Now let us analyze the crystals of orthorhombic and monoclinic symmetries. Here the coefficients $\pi_{44}$ and $\pi_{55}$ can easily be determined. As an example, we consider a $Y$-cut disk and assume that $n_{1} \simeq n_{3}=\bar{n}$ under the condition $X=R / 2$. The birefringence increment and the angle of optical indicatrix rotation are readily obtained from Eqs. (51) and (52):

$$
\begin{aligned}
& \delta\left(\Delta n_{13}\right)=-\frac{1}{2} \bar{n}^{3}\left[\left(\pi_{11}-\pi_{31}\right) \sigma_{1}(Z)+\left(\pi_{13}-\pi_{33}\right) \sigma_{3}(Z)\right], \\
& \tan 2 \zeta_{2}=\frac{2 \pi_{55} \sigma_{5}(Z)}{\left(\pi_{11}-\pi_{31}\right) \sigma_{1}(Z)+\left(\pi_{13}-\pi_{33}\right) \sigma_{3}(Z)} .
\end{aligned}
$$

The denominator of Eq. (78) is equal to the expression appearing in the square brackets of Eq. (77). Hence, solving the system of these equations would result in analytical expression of the coefficient $\pi_{55}$. The coefficient $\pi_{44}$ for the orthorhombic and monoclinic groups and the 
coefficient $\pi_{66}$ for the orthorhombic groups can be evaluated using respectively $X$ - and $Z$-cut disks (i.e., the systems of Eqs. (49) and (50) or Eqs. (53) and (54), respectively) and employing the same experimental procedures. As follows from Eqs. (55) and (56), it is impossible to evaluate the $\pi_{66}$ coefficient itself for the case of monoclinic crystals. Finally, the coefficients $\pi_{44}, \pi_{55}$ and $\pi_{66}$ for the triclinic crystals cannot be determined separately on the basis of our phenomenological relations (see Table 1).

\section{Conclusion}

In the present work we have rederived the main phenomenological relations that describe the changes occurring in the optical birefringence and the angle of optical indicatrix rotation of the crystalline disks loaded along their diameters. The relations are derived for all of the point symmetry groups. On this basis we have found that the piezooptic coefficients $\pi_{k k}(k=4,5,6)$ can be successfully determined for the crystals of almost all of the point groups. The corresponding technique is based on experimental studies of the optical anisotropy parameters induced along the chords of a crystalline disk compressed along its diameter. Only the crystals belonging to the triclinic system are problematic in this respect.

\section{References}

1. Narasimhamurty T S, Photoelastic and electrooptic properties of crystals. N. Y.: Plenum Press (1981).

2. Skab I, Smaga I, Savaryn V, Vasylkiv Yu and Vlokh R, 2011. Torsion method for measuring piezooptic coefficients. Cryst. Res. Technol. 46: 23-36.

3. Vasylkiv Y, Savaryn V, Smaga I, Skab I and Vlokh R, 2011. On determination of sign of the piezooptic coefficients using torsion method. Appl. Opt. 50: 2512-2518.

4. Krupych O, Savaryn V and Vlokh R, 2014. Precise determination of full matrix of piezo-optic coefficients with a four-point bending technique: the example of lithium niobate crystals. Appl. Opt. 53: B1-B7.

5. Savaryn V, Skab I, Krupych O and Vlokh R, 2012. The method for measuring piezooptic coefficients of crystals using a crystalline disk loaded along its diameter. Ukr. J. Phys. Opt. 13: $82-123$.

6. Frocht M M, Photoelasticity. N. Y.: John Wiley (1946).

7. Ajmera P K, Huner B, Dutta A K and Hartley C S, 1988. Simulation and observation of infrared piezobirefringent images in diametrically compressed semiconductor disks. Appl. Opt. 27: $752-757$.

Savaryn V., Krupych O., Skab I. and Vlokh R. 2014. Reinvestigation of piezooptic anisotropy appearing in a crystalline disk loaded along its diameter. Ukr.J.Phys.Opt. 15: 84-95.

Анотація. 3 метою виправлення помилок, присутніх у нашій попередній роботі (Savaryn V. et al. Ukr. J. Phys. Opt. 13 (2012) 82), ми повторно одержали основні феноменологічні співвідношення, що описують зміну оптичної анізотропії стиснутого вздовж діаметра кристалічного диска. На основі аналізу иих співвідномень представлено вдосконалений метод визначення п'єзооптичних коефіцієнтів $\pi_{44}, \pi_{55} i \pi_{66}$ для кристалів майже всіх груп симетріі. Метод базується на дослідженні просторового розподілу індукованих оптичного двозаломлення $і$ кута повороту оптичної індикатриси вздовж хорд $і$ діаметрів кристалічного диска, стиснутого вздовж його діаметра.

Ukr. J. Phys. Opt. 2014, Volume 15, Issue 2 